\title{
The effect of luminance decrements upon the detection of sinusoidal gratings
}

\author{
FREDERICK L. KITTERLE, E. MICHAEL BEASLEY, and JOSEPH BERTA \\ University of Toledo, Toledo, Ohio
}

\begin{abstract}
Observers detected 20-msec sinusoidal gratings presented at various asynchronies relative to a decrement in the mean luminance of a uniform field. Brief luminance decrements have no effect upon contrast thresholds determined with high-spatial-frequency gratings. With lowspatial-frequency gratings, transient threshold elevations were found at decrement onset and offset. For longer decrements, threshold changes were found for both high- and low-spatialfrequency gratings. However, the time courses of these changes differed. With high-spatialfrequency gratings, thresholds changed more gradually, whereas with low-spatial-frequency gratings, the time courses contained marked transients at the onset and offset of the decrement. These results are discussed in terms of contribution of multiple spatial-frequency channels to temporal resolution.
\end{abstract}

The human visual system is quite sensitive to brief luminance increments and decrements. The temporal response of the visual system to luminance increments has been determined by measuring thresholds for detecting a brief test flash presented at various times relative to the luminance increment (Crawford, 1947; see Boynton, 1972, for a review). Boynton and Kandel (1957) have referred to these data as "onresponse" records and suggest that they reflect receptive field activity. It may, in fact, be argued that these measurements characterize that temporal response of on-center receptive fields (Kuffler, 1953). It appears that the response of the visual system to luminance decrements may be signaled by a mechanism different from that signaling luminance increments.

Jung $(1961,1973)$ has proposed the existence of two visual systems: a B system, which responds to luminance increments and signals brighter, and a D system, which is sensitive to luminance decrements and signals darker. The B system is thought to reflect the activity of on-center off-surround receptive fields, and the D system, the activity of off-center on-surround receptive fields.

Recent research indicates that the temporal response of the B system may be an envelope reflecting the activity of a number of channels tuned to different spatial frequencies (Green, 1981). Green measured contrast thresholds for detecting gratings presented at various times relative to the onset of uniform field. Contrast thresholds for low-spatial frequency gratings were markedly elevated at the onset and offset of the field. With high-spatial-frequency gratings, contrast thresholds were found to be uni-

This research was supported by a Faculty Research Award to the first author from the Graduate School of the University of Toledo. The authors' mailing address is: Department of Psychology, University of Toledo, Toledo, $\mathrm{OH} 43606$. formly elevated for the entire duration of the flash. Green interpreted his results as indicating the existence of mechanisms with different impulse responses. Transient threshold elevations are assumed to reflect a mechanism with a biphasic response: an early excitatory component and a delayed inhibitory component (Kelly, 1971). The time course of threshold changes determined with high-spatial frequency gratings suggests a mechanism in which there is no delay between the center and the surround responses.

In contrast to experiments on luminance increments, there has been less work on the temporal response characteristics of the visual system to luminance decrements. Studies have compared the influence of flash duration (White, Irvin, \& Williams, 1980) and flicker rate (Magnussen \& Glad, 1975) upon the perception of darkness. Perceived darkness appears to be greater than perceived brightness, despite the fact that luminance decrements and increments may be of the same magnitude. Little is known about the way in which luminance decrements influence the sensitivity of the visual system, and there is no information about whether the response of the D system may also be considered as an envelope reflecting the activity of a number of underlying channels.

The present experiment was designed to determine the role of spatial-frequency channels in the detection of a luminance decrement. Sensitivity to an interruption in prevailing luminance was determined by measuring contrast thresholds for sinusoidal gratings presented at various times relative to a decrement in the luminance of a uniformly illuminated adapting field. The duration of the decrement was a variable in this study. The logic of the experiment is outlined below.

Consider the effect of a brief luminance decrement upon the detection of high-spatial-frequency gratings. Since temporal summation and response persis- 
tence is relatively long for channels detecting highspatial-frequency gratings, threshold should be the same when measured before, during, or after the decrement (Bowling \& Lovegrove, 1980; Breitmeyer, Levi, \& Harwerth, 1981; Legge, 1978). However, the same decrement should have a different effect upon the detection of low-spatial-frequency gratings. The lower critical duration and shorter response persistence of channels sensitive to low-spatial-frequency gratings suggests a greater sensitivity to rapid luminance fluctuations. Consequently, visual thresholds are expected to depend upon when the grating is presented relative to the luminance decrement. On the basis of other research, transient threshold elevations are expected at the onset and offset of the decrement with these stimuli (Green, 1981; Kitterle, Corwin, \& Berta, 1979; Mitov, Vassilev, \& Manahilov, 1981).

\section{METHOD}

\section{Observers}

Two observers, E.M.B. (30 years) and J.B. (28 years) participated in this experiment. Both had normal or corrected-tonormal visual acuity (J.B.) and were experienced psychophysical observers.

\begin{abstract}
Apparatus
A two-channel optical system was used to present the stimuli. One channel of the system (the test channel) was used to present the test stimuli, which were sinusoidal gratings generated on an oscilloscope (Tektronix 5103 D/N, P-31 phosphor) using standard techniques. The test stimuli were presented as 20 -msec flashes modulated about the mean luminance of the oscilloscope $(10 \mathrm{~cd} /$ $\mathrm{m}^{2}$ ). The second channel (the mask channel) was illuminated by a projector whose light was adjusted by means of color filters to match the oscilloscope in color. Neutral density filters (Wratten No. 96) reduced the luminance of this channel to $40 \mathrm{~cd} / \mathrm{m}^{2}$. A shutter in this channel controlled the duration of the luminance decrement. Aperatures in both channels created circular displays that, at a viewing distance of $86 \mathrm{~cm}$, subtended $6 \mathrm{deg}$.

The light paths of both channels were superimposed by means of a beamsplitter to produce a single uniform circular display whose total luminance varied from $50 \mathrm{~cd} / \mathrm{m}^{2}$ when both fields were exposed to $10 \mathrm{~cd} / \mathrm{m}^{2}$ when the mask channel was extinguished. This drop in luminance constituted the luminance decrement. The observers viewed the display monocularly with the right eye. A chinrest was used to stabilize viewing. The timing of the test flashes, luminance decrements, and SOAs was controlled by a microprocessor (Automated Data Systems 1800E).
\end{abstract}

\section{Procedure}

At the beginning of the experimental session, the observers adapted to the mean luminance of the combined fields for 5 min. Following the adaptation period, resting thresholds were determined. On these trials, the test gratings were flashed and the observer used the method of adjustment to set the gratings to threshold by means of a 10-turn potentiometer. Five threshold determinations were made. There was a 2-sec intertrial interval between flashes. After the resting thresholds were obtained, test trials commenced. On test trials, the mask channel was decremented for a given duration. The test flash was presented at a randomly chosen onset asynchrony relative to the decrement, and the threshold for detecting it was determined. Contrast thresholds were determined from the following expression:

$$
C=\frac{L \max -L \min }{L \max +L \min }
$$

Following the luminance decrement, the masking field was reilluminated and the observers readapted for $20 \mathrm{sec}$ before the next test presentation. Once the observer made a threshold determination for a given SOA, a new one was chosen randomly and the test-decrement sequence was repeated until five replications had been made at each SOA. Five additional resting thresholds were determined at the end of the test session. The duration of the luminance decrements and the spatial frequency of the test gratings were chosen randomly and varied across sessions.

\section{RESULTS}

The results of this experiment are presented in Figures 1A, 1B, 1C, and 1D for Observers J.B. (open circles) and E.M.B. (filled circles). In these figures, contrast thresholds relative to the resting threshold are plotted as a function of SOA. Each figure shows the results for different durations of the mask decrement. The parameter of each figure is the spatial frequency of the test grating. For clarity, the curves for the 1.4-, 3.5-, and 5.25-cycle/deg (cpd) gratings are shifted upwards. In general, there is rather good agreement between observers.

Consider first the results obtained with the briefest decrement utilized. Notice that when the duration of the decrement is $20 \mathrm{msec}$, most of the fluctuation in threshold is found with the 0.7-cpd target. A somewhat smaller change in threshold is suggested in the data obtained with the 1.4-cpd target. In contrast, the thresholds for the 3.5- and 5.25-cpd targets are not influenced by a luminance decrement of this duration.

With the 60-msec decrement (Figure 1B), the data for the 0.7- and 1.4-cpd targets again show transient threshold elevations at the onset and offset of the decremental flash. However, the magnitude of the transient obtained with the 0.7 -cpd grating is considerably larger for J.B. than for E.M.B. A comparison of the results obtained, with the 0.7-cpd grating, at $20 \mathrm{msec}$ with those obtained at $60 \mathrm{msec}$ suggest summation of the on and off transients at the shorter duration. There is a small, gradual change in threshold with the 3.5-cpd target and no evidence of transients at either onset or offset of the luminance decrement.

In Figures $1 \mathrm{C}$ and 1D, the results for the 100- and $500-\mathrm{msec}$ decrements are summarized. With a $100-$ msec decrement, transient activity is evident around the onset of the decrement for all but the 5.25-cpd target. By 500 msec (Figure 1D), there are clear changes in threshold for all test targets, but they are more gradual for the 3.5- and 5.25-cpd gratings than for the 1.4- and 0.7-cpd targets. In addition, there is some evidence of a small transient elevation of threshold with the 3.5-cpd grating in the data of $\mathrm{Ob}$ server J.B. at the onset 500 -msec decrement (see Figure 1D). This transient is not evident in the results obtained with the 5.25-cpd grating for either observer. Transient contrast threshold elevations are also not found with increments in uniform field lumi- 


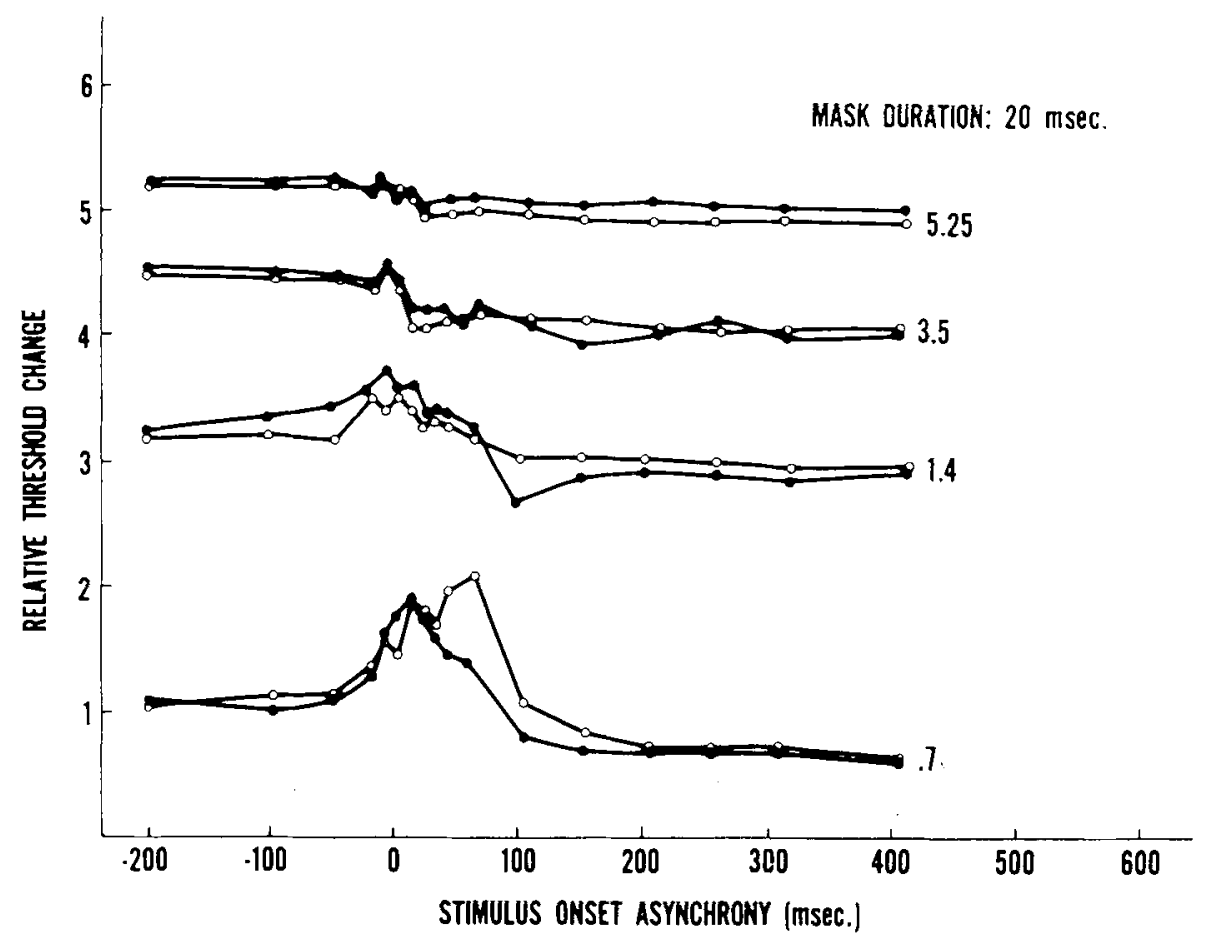

Figure 1A. Relative threshold change plotted as a function of stimulus onset asynchrony (SOA) for a decrement duration of 20 msec. The number beside each set of data refers to the spatial frequency of the test grating. The data for the 1.4-, 3.5-, and 5.25-cpd gratings have been vertically shifted for clarity. Results for Observers J.B. (open circles) and E.M.B. (filled circles).

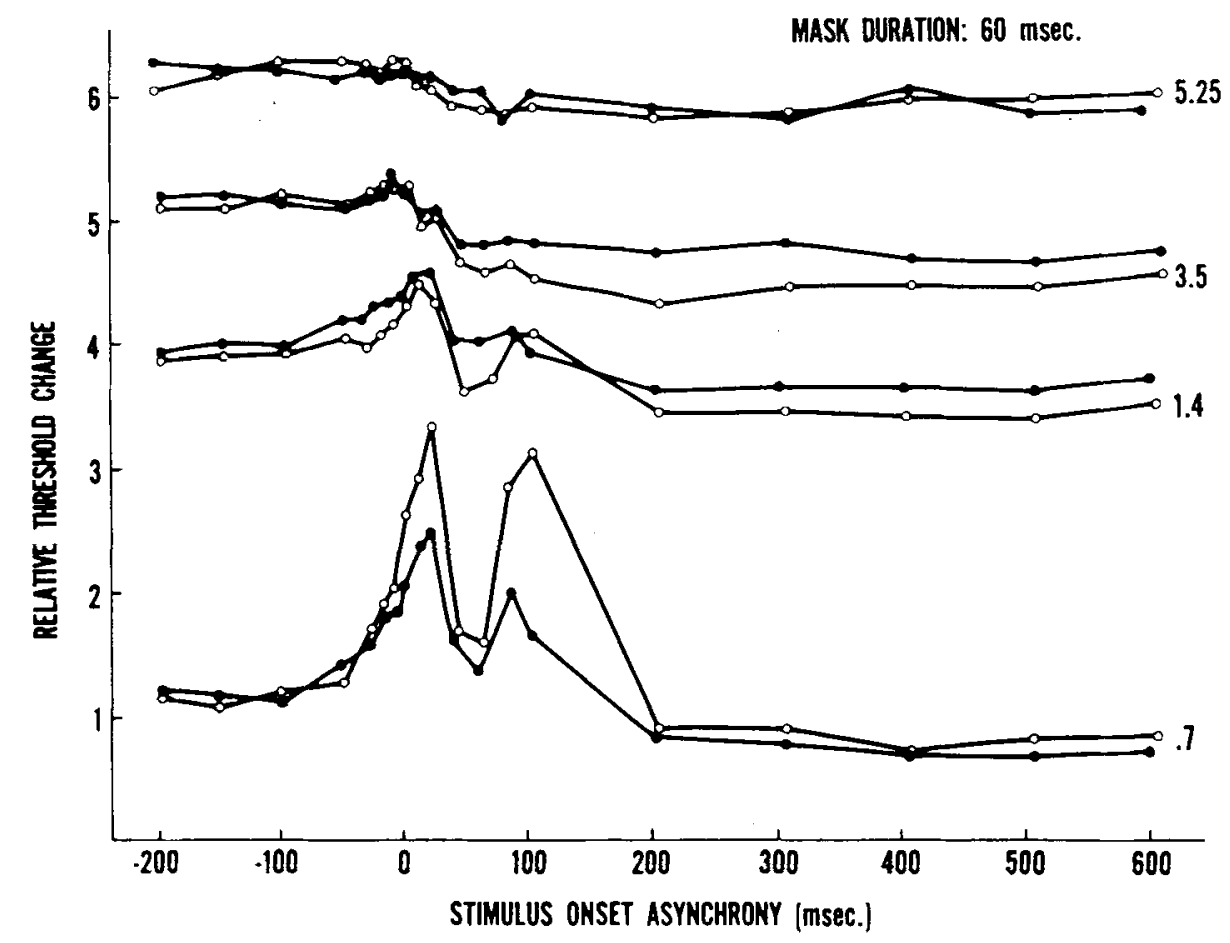

Figure 1B. Same as Figure 1A for a decrement of 60 msec. 


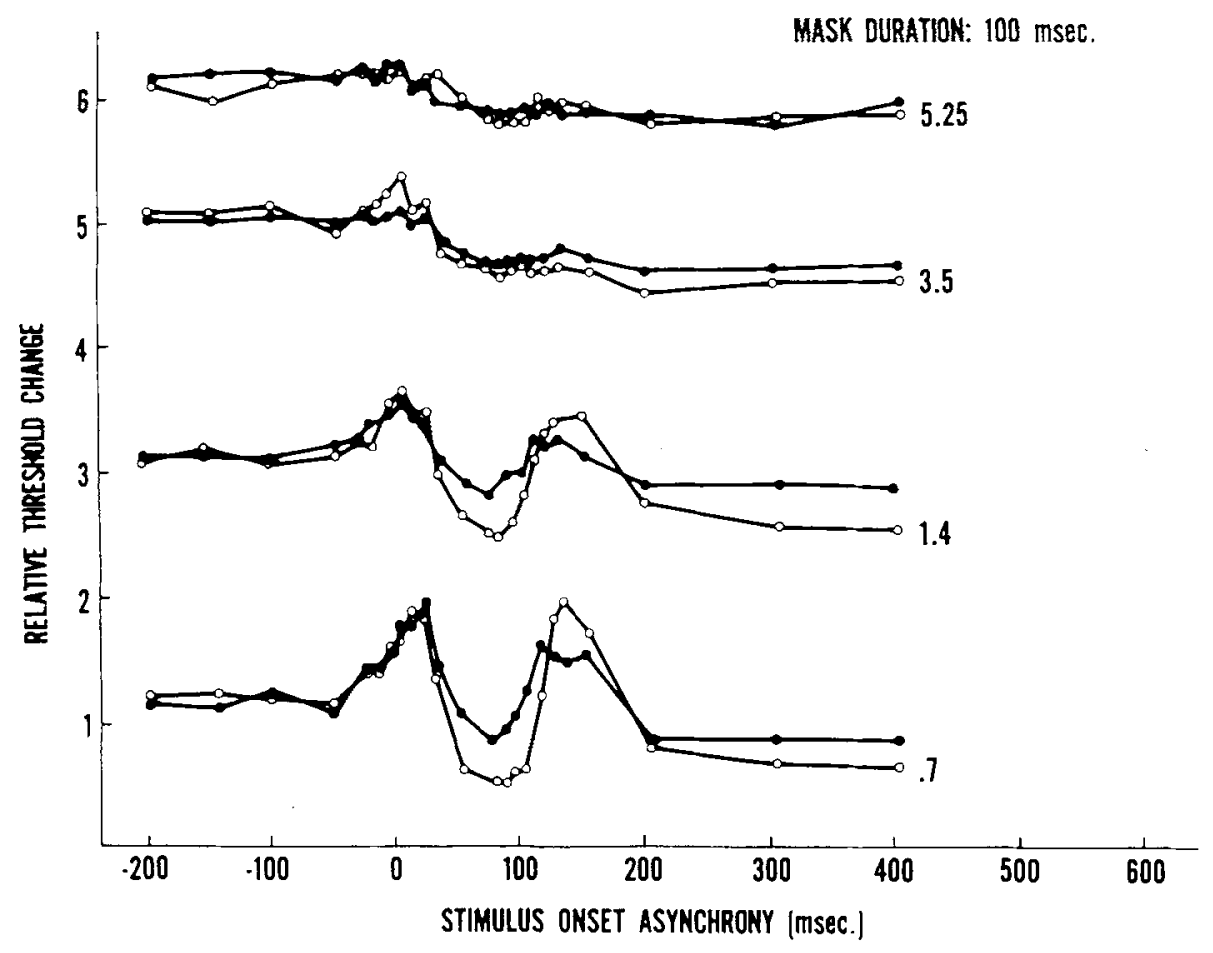

Figure 1C. Same as Figure 1A for a decrement of $100 \mathrm{msec}$.

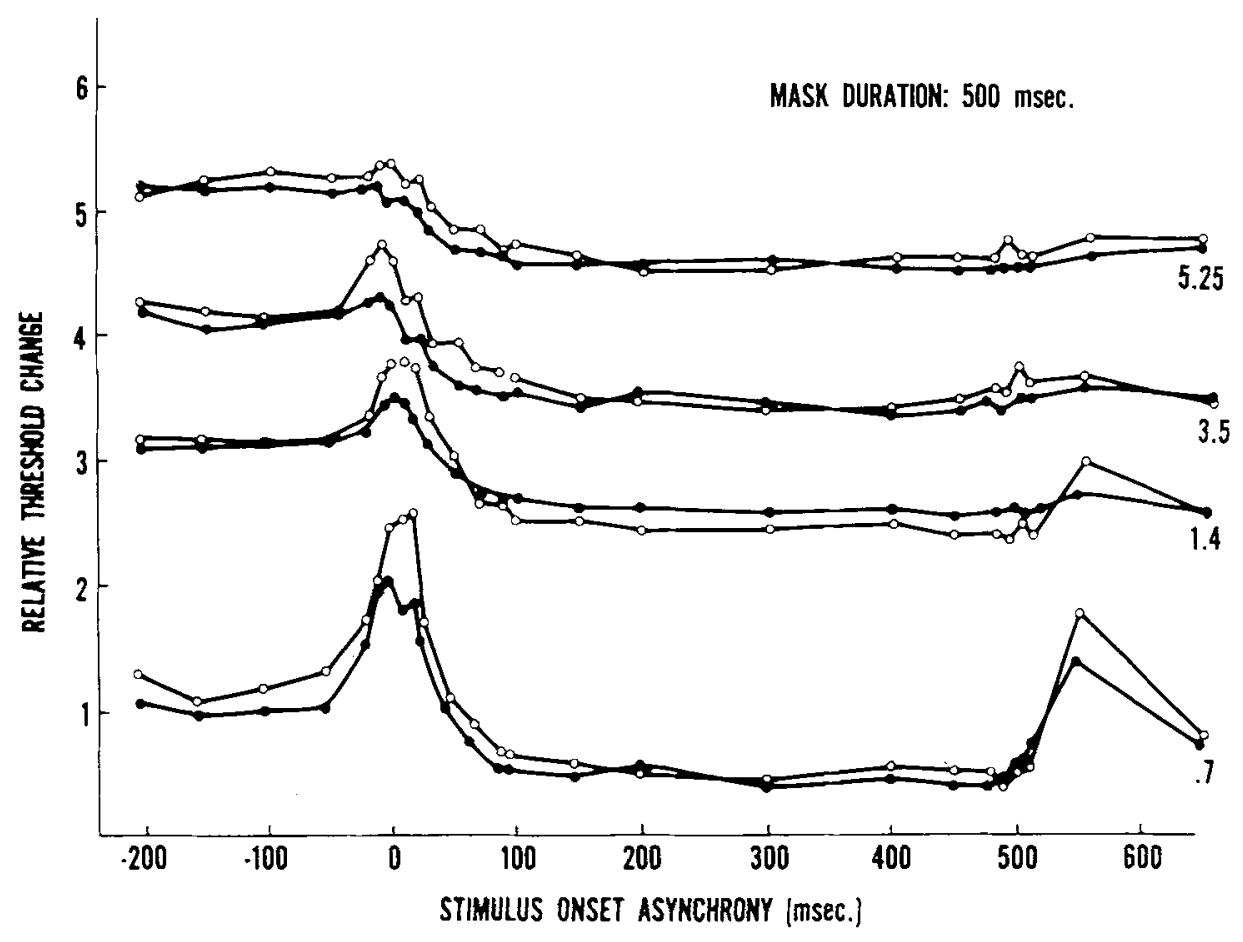

Figure 1D. Same as Figure 1A for a decrement of 500 msec. 
nance with high-spatial-frequency gratings (Kitterle et al., 1979; Green, 1981).

In Figures $2 \mathrm{~A}, 2 \mathrm{~B}$, and $2 \mathrm{C}$, the data have been replotted for the 0.7-, 1.4-, and 5.25-cpd test probes respectively. In these figures, the parameter of the curves is the duration of the luminance decrement. These figures make it easier to compare the effects of decrement duration on temporal changes in threshold for a given spatial frequency.

In Figure 2A, the effects of duration are shown for the 0.7-cpd grating. Across all durations, transient threshold elevations are readily seen at onset and offset. Notice, however, that the magnitude of this effect appears to change in a nonmonotonic fashion with duration for both the onset and the offset transients. The flash duration producing the maximum elevation in threshold is found at $60 \mathrm{msec}$. The results obtained with the 1.4-cpd grating also show distinct transients for flash durations greater than $20 \mathrm{msec}$ (Figure 2B). The magnitude of these transients is, however, smaller than that found with the 0.7-cpd grating, and the magnitude appears to change in a monotonic fashion with duration. The data obtained with the 5.25-cpd grating (Figure 2C) differ considerably from the data described earlier. First, the changes in threshold are considerably more gradual and have a smaller magnitude. Second, there is virtually no evidence in the results of either observer of any marked transient activity accompanying decrement onset or offset. Finally, it is apparent that there is little, if any, effect on threshold for flash decrements less than 100 msec.

\section{DISCUSSION}

The results of the present experiment indicate that sensitivity to a luminance decrement depends upon two factors, the duration of the luminance decrement and the spatial frequency of the target used to measure changes in sensitivity. For example, threshold changes are not found for flash decrements of 20-100 msec when a 5.25-cpd test grating is used. It appears that the visual system is not sensitive to flashes in this range. Notice, however, the marked transient fluctuations in threshold over this range when a 0.7-cpd target is used. The changes are found primarily at the onset and offset of the decrement. Thus, brief luminance decrements influence contrast thresholds of low, but not of high, spatial frequency gratings. The visual system appears to be sensitive to decrements at longer durations regardless of the test spatial frequency. But it is evident that the time

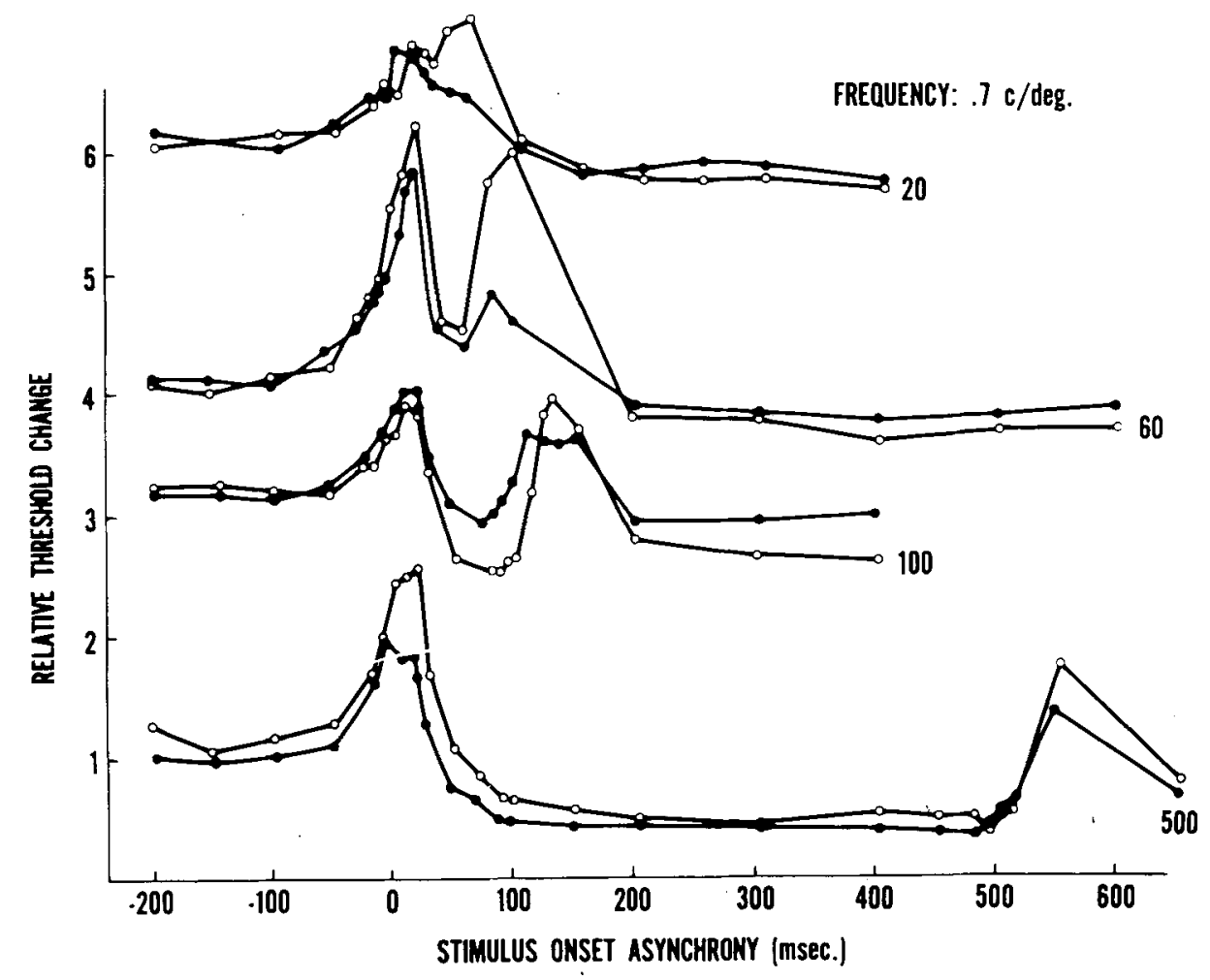

Figure 2A. Relative threshold change plotted as a function of SOA for a 0.7-cpd sinusoidal grating. The numbers to the right of each set of data are for a particular decrement duration. The data for the 20-, 60-, and 100 -msec durations have been shifted vertically for clarity. The results are shown for Observers J.B. (open circles) and E.M.B. (filled circles). 


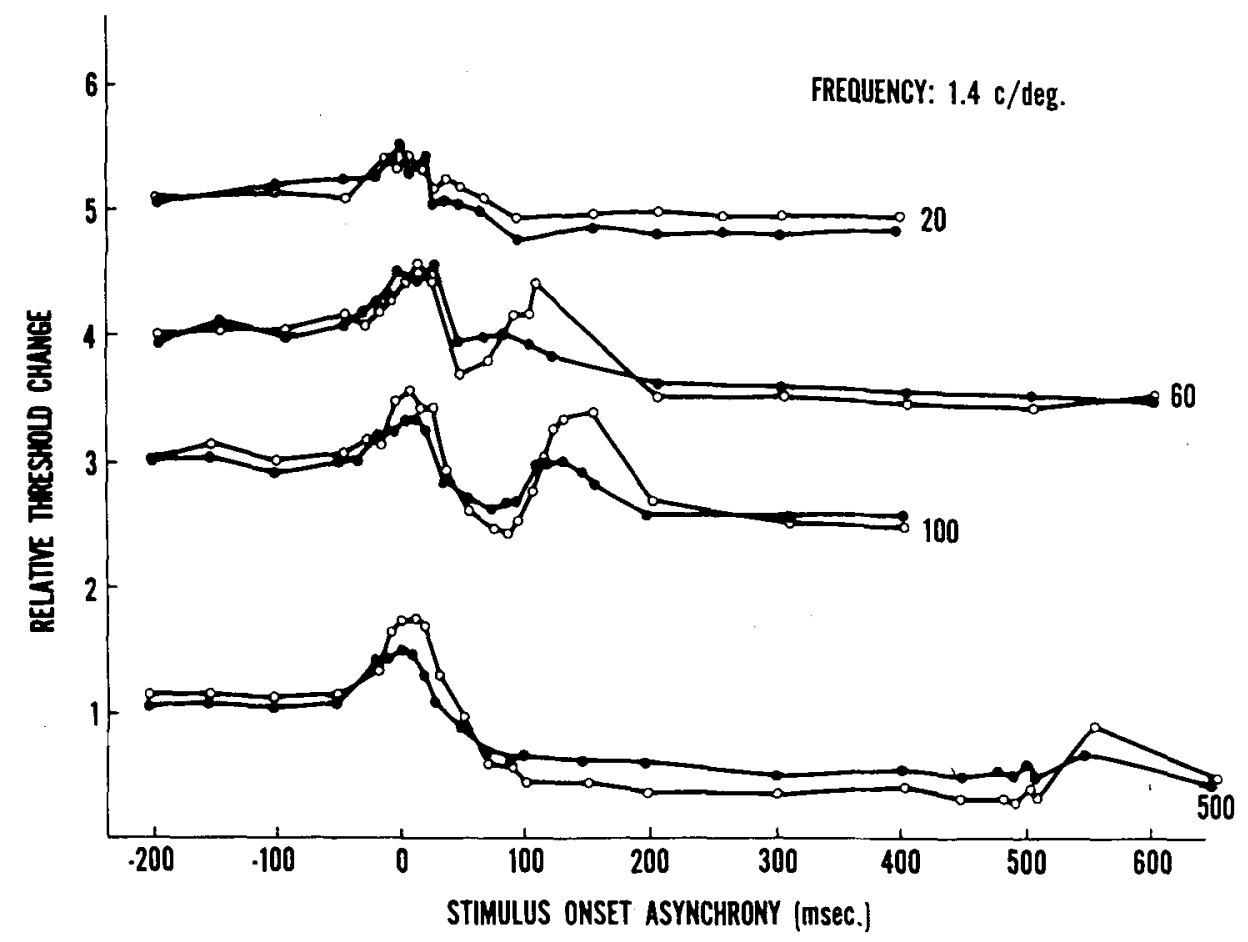

Figure 2B. Same as Figure 2A for a 1.4-cpd grating.

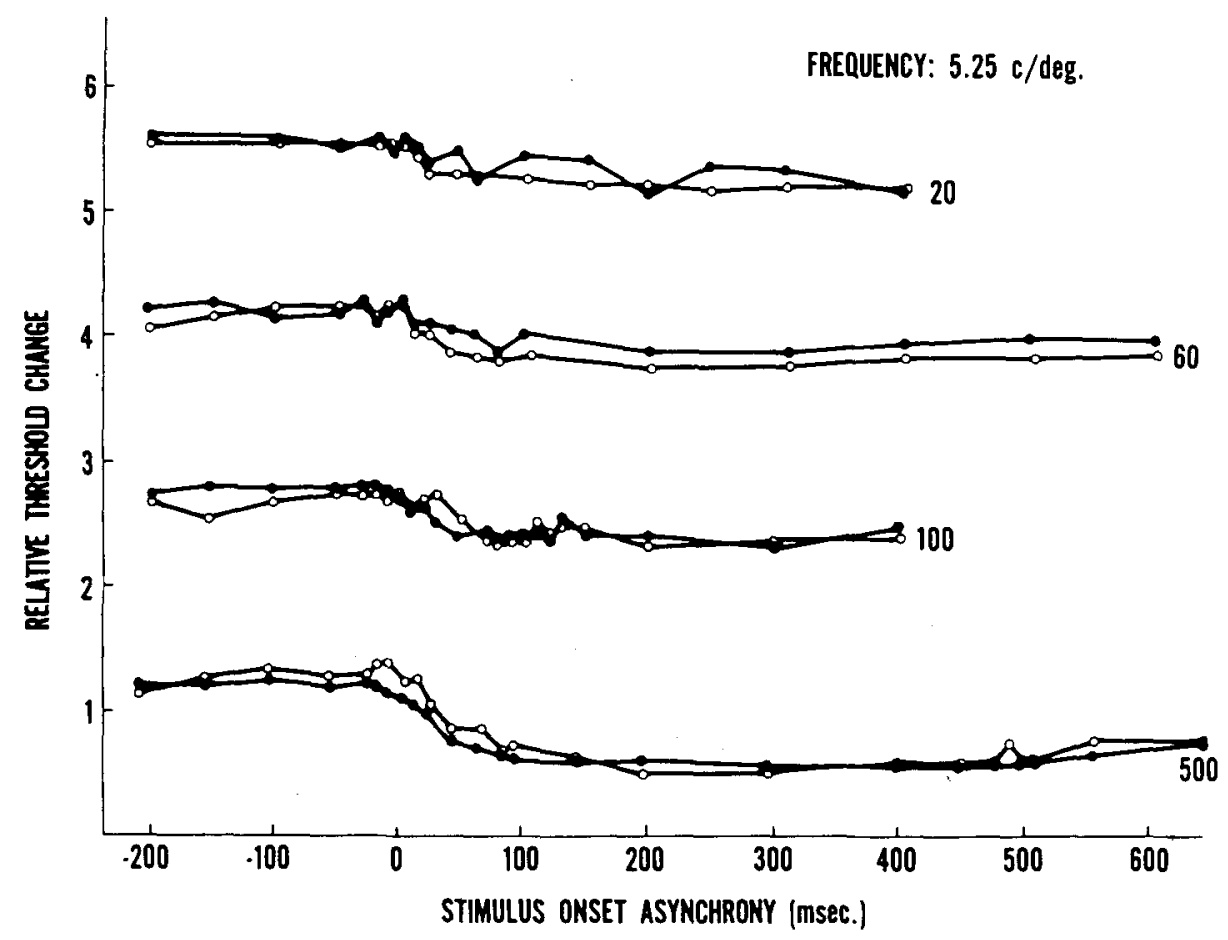

Figure 2C. Same as Figure 2A for a 5.25-cpd grating. 
course of changes in threshold depend upon the spatial frequency of the test grating. Changes in threshold become more gradual, and the transients associated with the decrement onset and offset are progressively reduced with increases in spatial frequency.

The differential effects of target spatial frequency upon visual threshold are consistent with the interpretation that the D system, like the B system, is also composed of a number of channels, each tuned to a different range of spatial frequencies. For example, when the luminance decrement is very brief, it may fail to have an effect on channels responding to high spatial frequencies because these mechanisms appear to have longer response latencies than those detecting low spatial frequencies (Breitmeyer, 1975; Vassilev \& Mitov, 1967). In addition, the decrement in luminance may fail to be perceived, because the activity of high-spatial-frequency channels, which respond to the prevailing level of luminance, may not abruptly cease when the luminance is decremented. Indeed, research indicates that there is an increase in visual persistence with an increase in target spatial frequency (Bowling \& Lovegrove, 1980; Bowling, Lovegrove, \& Mapperson, 1979; Meyer \& Maguire, 1977). Finally, brief decrements in luminance may fail to have an effect upon the visual system because of the longer limits of temporal summation for high-spatial-frequency channels (Legge, 1978; Nachmias, 1967). This study complements that of Corfield, Frosdick, and Campbell (1978), who found that the detection of an interruption or discontinuity in the presentation of sinusoidal gratings depended upon the spatial frequency of the gratings. As the spatial frequency of the gratings increased, the duration of the discontinuity had to be correspondingly increased in order to be detected.

The present results may have important implications for related work involving temporal discriminations. Estimates of visual persistence can differ by 70-100 msec, depending upon the choice of criteria employed by the observer (Allan, 1975; Sternberg \& Knoll, 1973). Erwin (1976) showed the persistence was longer if the observer had to identify the stimulus as well as report its phenomenal offset than if he/she was simply to report its offset. Criterion effects may reflect, in part, the differential weighting of the output spatial frequency channels. For example, in the study by Erwin, the identification task may have increased the weight given to the mechanisms responsive to the high-spatial-frequency Fourier components in the target. These channels have been shown to yield longer persistence (Meyer \& Maguire, 1977). A similar explanation may account for the finding that the limits of temporal summation are shorter when a brightness criterion, rather than a form criterion, is used (Kahneman \& Norman, 1964).
Adaptation studies provide additional support for this idea. Flicker adaptation decreases the sensitivity of channels for detecting low-spatial-frequency targets (Green, 1981). On the basis of the present hypothesis, this should have the effect of shifting processing to the higher spatial-frequency channels, and, as a result, temporal resolution should decrease. This prediction has been verified by Breitmeyer et al. (1981).

In summary, the results of the present experiment support the view that the response of the visual' system to a decrement in luminance may be an envelope reflecting the activity of a number of spatially tuned mechanisms. Criterion effects in temporal resolution may reflect, in part, differences in the weight given to the output of these mechanisms.

\section{REFERENCES}

Allan, L. G. (1975). Temporal order psychometric functions based on confidence ratings. Perception \& Psychophysics, 18, 369-372.

Bowling, A., \& Lovegrove, W. (1980). The effect of stimulus duration on the persistence of gratings. Perception \& Psychophysics, 27, 574-578.

Bowling, A., Lovegrove, W., \& Mapperson, B. (1979). The effect of spatial frequency and contrast on visual persistence. Perception, 8, 529-539.

Boynton, R. M. (1972). Discrimination of homogeneous double pulses of light. In L. Hurvich (Ed.), The handbook of sensory physiology (Vol. 7/4, pp. 202-232).

Boynton, R. M., \& Kandel, G. R. (1957). On-responses of the human visual system as a function of adaptation level. Journal of the Optical Society of America, 47, 275-286.

Breitmeyer, B. G. (1975). Simple reaction time as a measure of temporal response properties of transient and sustained channels. Vision Research, 15, 1411-1412.

Brettmeyer, B. G., Levi, D. M., \& HaRWeth, R. S. (1981). Flicker masking in spatial vision. Vision Research, 21, 13771385.

Corfield, R., Frosdick, J. P., \& Campbell, F. W. (1978). Grey-out elimination: The role of spatial waveform. Vision Research, 18, 1305-1311.

CRAwFond, B. H. (1947). Visual adaptation in relation to brief conditioning stimuli. Proceedings of the Royal Society (London), B 134, 283-302.

Erwin, D. E. (1976). Further evidence for the two components in visual persistence. Journal of Experimental Psychology: Perception and Human Performance, 2, 191-209.

GreEN, M. (1981). Spatial frequency effects in masking by light. Vision Research, 21, 861-866.

JuNa, R. (1961). Neuronal integration in the visual cortex and its significance for visual information. In W. A. Rosenblith (Ed.), Sensory Communication. New York: M.I.T. Press.

Jung, R. (1973). Visual perception and neurophysiology. In R. Jung (Ed.), Handbook of sensory physiology: Central visual information (Vol. 7/3A). New York: Springer.

Kahneman, D., \& Norman, J. The time-intensity relation in visual perception as a function of observer's task. Journal of Experimental Psychology, 68, 215-220.

KELLY, D. H. (1971). Theory of flicker and transient responses. I. Uniform flashes. Journal of the Optical Society of America, 61, 537-546.

Kitterle, F. L., Conwin, T. R., \& Berta, J. (1979). Masking of sinusoidal targets by uniform fields of unequal duration. Journal of the Optical Society of America, 69, 1445. 
Kuffler, S. W. (1953). Discharge patterns and functional organization of mammalian retina. Journal of Neurophysiology, 16, 37-68.

LEGGE, G. E. (1978). Sustained and transient mechanisms in human vision: Temporal and spatial properties. Vision Research, 18, 69-81.

Magnussen, S., \& Glad, A. (1975). Brightness and darkness enhancement during flicker: Perceptual correlates of neuronal B- and D-systems in human vision. Experimental Brain $\boldsymbol{R e}$ search, 22, 399-413.

Meyer, G. E., \& Maguire, W. (1977). Spatial frequency and the mediation of short-term storage. Science, 198, 524-525.

Mitov, D., Vassilev, A., \& Manahilov, V. (1981). Transient and sustained masking. Perception \& Psychophysics, 30, 205-210.

Nachmias, J. (1967). The effect of exposure duration on visual contrast sensitivity with square-wave gratings. Journal of the Optical Society of America, 57, 421-427.

Ste RnBerg, S., \& KNoll, R. L. (1973). The perception of temporal order: Fundamental issues and a general model. In $\mathrm{S}$. Kornblum (Ed.), Attention and performance (Vol. 4). New York: Academic Press.

Vassilev, A., \& Mitov, D. (1967). Perception time and spatial frequency. Vision Research, 16, 89-92.

White, T. W., Irvin, G. E., \& Williams, M. C. (1980). Asymmetry in the brightness and darkness Broca-Sulzer Effects. Vision Research, 20, 723-726.

(Manuscript received April 4, 1983; revision accepted for publication November 5,1983 .) 